\title{
USP15 inhibits HPV16 E6 degradation and catalytically inactive USP15 has reduced inhibitory activity
}

\author{
Y. YAGINUMA*, M. YOSHIMOTO*, A. TOKUDA
}

\begin{abstract}
Department of Oncology, Graduate School of Health Sciences, Faculty of Life Sciences, Kumamoto University, 4-24-1 Kuhonji, Chuouku, Kumamoto 862-0976, Japan
\end{abstract}

Received June 19, 2017; revised November 2, 2017; accepted April 5, 2018

\begin{abstract}
Summary. - High-risk human papillomaviruses (HPVs) possess transforming activity leading to development of the cancer, including oropharyngeal, anal, penile, vulvar, vaginal, and cervical cancer. The stability of E6 is essential for its complete function as an oncoprotein. Using the yeast two-hybrid system, we identified ubiquitin-specific protease 15 (USP15) as an HPV16 E6-interacting protein. USP15 cleaves polyubiquitin chains of HPV16 E6 and/or ubiquitin precursors. Our results indicate that USP15 could increase the level of HPV16 E6 by inhibiting E6 degradation. USP15 inhibited the degradation of HPV16 E6 in dose-dependent manner. In contrast, catalytically inactive mutants of USP 15 had a reduced inhibitory effect on E6 degradation. In particular, USP15 mutants of all three cysteine boxes and the NHL mutant of the KRF box had a drastically reduced inhibitory effect on HPV16 E6 degradation. In addition, HPV16 E6 mRNA was not induced by USP15; therefore, HPV16 E6 appears to be post-translationally regulated. These results suggest that USP15 has the ability to stabilize E6 as a deubiquitinating enzyme, and as an oncoprotein affects biological functions in infected human cells.
\end{abstract}

Keywords: cervical cancer; HPV; E6; USP15; carcinogenesis

\section{Introduction}

Human papillomaviruses (HPVs) have been etiologically linked to human cervical cancer (Strohl et al., 2015). HPVs target skin or mucosal epithelial cells (zur Hausen, 1991a,b). HPVs can be classified as either high-risk or low-risk depending on the transforming potential of the virus. Most cervical cancers and a significant proportion of other anogenital cancers have been associated with infection by a small number of high-risk genital HPV types (Dell and Gaston, 2001).

Tumorigenesis induced by high-risk genital HPVs has been linked to the expression of two viral oncoproteins, E6

E-mail: yaginuma@kumamoto-u.ac.jp; phone: +81-96-373-5491. "These two authors contributed equally to this work.

Abbreviations: $\mathrm{DUB}=$ deubiquitinating enzyme; FOA = fluorouracil; HPVs = human papillomaviruses; hTERT = human telomerase reverse transcriptase; $\mathrm{MDM} 2$ = murine double minute 2; SC = synthetic complete dropout medium; USP = ubiquitinspecific protease and E7, which cooperate in cellular immortalization and transformation processes (Schwarz et al., 1985). An important function of the high-risk type E6 is the degradation of p53 (Crook et al., 1991; Crook et al., 1994; Pim and Banks, 1999; Scheffner et al., 1993; Thomas et al., 1999; Yang and Lu, 2015) via E6-AP (Alarcon et al., 1999; Ansari et al., 2012; Beaudenon and Huibregtse, 2008; Cooper et al., 2003). The degradation of $\mathrm{p} 53$ by HPV E6 is specific to high-risk HPVs, but several studies have suggested that low-risk type E6 may be able to bind with low affinity to p53 (Kehmeier et al., 2002; Li and Coffino, 1996; Oh et al., 2004; Stewart et al., 2004; White et al., 2012). High-risk type E6 is also known to activate telomerase via the upregulation of hTERT expression (Liu et al., 2008; Van Doorslaer and Burk, 2012; Wang et al., 2015a,b; Xu et al., 2013). Many cellular proteins have already been reported to bind to high-risk HPV E6 proteins, such as p300/CREB-binding protein (CBP) (Howie et al., 2011; Xie et al., 2014), the focal adhesion protein paxillin (Vande Pol et al., 1998; Wu et al., 2014), the calcium-binding protein E6-binding protein (E6-BP) (Sherman et al., 2002), and 
PDZ domain proteins, but the physiological importance of these interactions with E6 proteins remains unknown. In this study, to analyze the precise function of HPV16 E6, we used the yeast two-hybrid system to investigate cellular proteins that interact with HPV16 E6. We found ubiquitinspecific protease 15 (USP15) to be a protein that interacts with HPV16 E6. USP15 encodes a protein that consists of 952 amino acids and functions as a deubiquitinating enzyme (DUB). Vos et al. (2009) already proved by mass spectrometry analysis that USP15 could bind to HPV16 E6.

Deubiquitination is an essential process that releases ubiquitin chains from ubiquitin-protein conjugates. The human genome encodes approximately 100 putative DUBs that belong to a superfamily of proteases. Within the DUB family, USP (ubiquitin specific protease), and ubiquitin C-terminal hydrolase are the most well-characterized enzymes. USP is the major family of a variety of DUBs (Clague et al., 2012; Kim et al., 2003). USP proteins constitute the largest family with more than 60 members and they belong to the cysteine protease family. A hallmark of this class of enzymes is the presence of a catalytic core displaying the so-called histidine (His) and cysteine (Cys) boxes. Several recent examples from disparate model systems indicate that specific DUBs can indeed target specific proteins and that these deubiquitination events can have a major physiological impact (Baker et al., 1992; Kawakami et al., 1999).

A key event of HPV-induced oncogenesis is the integration of viral DNA into the genome of the infected cells, accompanied by the disruption of the viral E2 DNA region and over-expression of the viral E6 and E7 oncoproteins (Eichten et al., 2004). HPV16 E6 is an oncoprotein of which its up-regulation is needed during HPV-related carcinogenesis. In this study, we confirmed that HPV16 E6 could bind to USP15, which is a member of the UBP family of DUBs (Baker et al., 1999). We speculated that USP15 stabilized HPV16 E6 protein by binding with each other after the infection to human cells. This stabilization of E6 appears to be important for its function as an oncoprotein in the infected human cells.

\section{Materials and Methods}

Cell lines, transfection and construction of plasmids. 293T and SiHa cells were obtained from the American Type Culture Collection (Manassas, USA). Cells were grown in Dulbecco's modified Eagle's medium supplemented with $10 \%$ fetal bovine serum and antibiotics. Transient transfection was carried out using Lipofectamine LTX reagents (Invitrogen, USA). HA-tagged HPV E6 and FLAG-tagged USP15 expression plasmids were constructed by cloning of HPV16 E6 DNA and the human USP15 cDNA into pCMV-HA (BD Science, USA) and pFLAG-CMV-2 (Sigma, USA) expression vectors, respectively. The E6 DNA of HPV16 was gener- ated by PCR using plasmids (pHPV16) obtained from the Japanese Collection of Research Bioresources Gene Bank (National Institute of Biomedical Innovation, Osaka, Japan). The primer sequences for the construction of these expression vectors are summarized in Table 1.

Yeast two-hybrid assay. The yeast two-hybrid assay was performed by the sequential method using the Proquest two-hybrid system (Invitrogen) according to the manufacturer's instructions. Briefly, HPV16 E6 DNA was cloned into the shuttle vector pDEST32 using the Gateway system (Invitrogen) and the vector was then transfected into MaV203 yeast cells to establish a yeast strain harboring the pDEST32-HPV16 E6 bait plasmid. The human fetal brain cDNA library (Invitrogen) was cloned into vector pPC86 and then transfected into HPV16 E6-competent yeast cells. Plasmids from the positive clones were isolated, and the interaction phenotypes were assessed using various plates. Control yeast strains supplied in the Proquest two-hybrid system were used to assess the strength of the interactions, which were designated A-E as follows: $\mathrm{A}=$ no interaction; $\mathrm{B}=$ weak interaction; $\mathrm{C}=$ moderate interaction; $\mathrm{D}=$ strong interaction; and $\mathrm{E}=$ very strong interaction. Control A contained no inserts in the resident plasmids; control B represented the interaction between human $\mathrm{Rb}$ and human E2F1; control $\mathrm{C}$ represented the interaction between drosophila DP and drosophila E2F; control D represented the interaction between rat cFos and mouse cJun; and control E contained GAL4 in pCL1 and empty pPC86 vector. The PCR primer sequences used in the yeast two-hybrid assay are shown in Table 1.

Site-directed mutagenesis. Site-directed mutagenesis was performed using the Gene Tailor ${ }^{\mathrm{TM}}$ site-directed mutagenesis system (Invitrogen). The primer sequences used in the construction of the mutant pFLAG-CMV2-USP15 vectors are shown in Table 1 . In total, we constructed 12 USP 15 mutants (Table 2), including seven mutants of the Cys box, three mutants of the His box, and one mutant each of the aspartic acid (Asp) domain and the KRF domain.

Western blot and co-immunoprecipitation. Proteins were extracted using elution buffer containing $150 \mathrm{mmol} / \mathrm{l} \mathrm{NaCl}$, $50 \mathrm{nmol} / \mathrm{l}$ Tris ( $\mathrm{pH} 8.0$ ), and Halt ${ }^{\mathrm{TM}}$ protease inhibitor cocktail (EDTA-free; Thermo Fisher Scientific, USA) and homogenizing in a Sonifier 450 (Branson, Japan). For Western blot, the cell lysates were resolved by $10 \%$ SDS-PAGE and transferred onto nitrocellulose membranes. The membranes were then incubated with primary antibodies, then with peroxidase-conjugated speciesmatched secondary antibodies, and developed using ECL-Plus (GE Healthcare, USA). Anti-FLAG M2 (Sigma), anti-HA (Roche, Germany), anti-USP15 (ab97533; Abcam, UK), anti- $\beta$-actin (A5316; Sigma), anti-HPV16 E6 (sc-1583; Santa Cruz Biotechnology, USA), and anti- $\beta$-tubulin (GTX101279; GeneTex, USA) antibodies were used. Reciprocal co-immunoprecipitation was performed in $293 \mathrm{~T}$ cells transfected with the plasmids containing pCMV-HA-HPV16 E6 and pFLAG-CMV2-USP15 constructs using anti-FLAG magnetic beads (Sigma) and anti-HA magnetic beads (Thermo Scientific, USA). After Western blot, anti-HA precipitation was developed by anti-USP15 antibody and anti- 
Table 1. Primers used for cloning, mutagenesis and quantification

\begin{tabular}{|c|c|c|}
\hline Target & Name & Sequence \\
\hline \multicolumn{3}{|c|}{ Primer sequences for the construction of Gate Way cloning } \\
\hline \multirow{2}{*}{ HPV 16 E6 DNA } & attB1 & GGGGACAAGTTTGTACAAAAAAGCAGGCTTTATGCACCAAAAGAGAACTGC \\
\hline & attB2 & GGGGACCACTTTGTACAAGAAAGCTGGGTACAGCTGGGTTTCTCTACGTGT \\
\hline \multicolumn{3}{|c|}{ Primer sequences for the construction of expression vector } \\
\hline \multirow{2}{*}{ HA-HPV 16 E6 } & forward & GACCGGAATTCTGTTTCAGGACCCACAGGAG \\
\hline & reverse & CAGGAAGATCTTTACAGCTGGGTTTCTCTAC \\
\hline \multirow{2}{*}{ FLAG-USP15 } & forward & TACCCAAGCTTGCGGAAGGCGGAGCGGCGGATC \\
\hline & reverse & TCTAGTCTAGATTAGTTAGTGTGCATACAGT \\
\hline \multicolumn{3}{|c|}{ Primer sequences for site directed mutagenesis of USP15 cysteine box } \\
\hline \multirow{2}{*}{$1^{\text {st }}$ Cys $\rightarrow$ Ser } & forward & ACAATGAACAGCCAGGCCTCTCTGGCCTAAGTAA \\
\hline & reverse & GAGGCCTGGCTGTTCATTGTTTCTTCCAGG \\
\hline \multirow{2}{*}{$2^{\text {nd }}$ Cys $\rightarrow$ Ser } & forward & TAAGTAACTTGGGAAATACGTCTTTCATGAACTC \\
\hline & reverse & CGTATTTCCCAAGTTACTTAGGCCACAGAGG \\
\hline \multirow{2}{*}{$3^{\text {rd }}$ Cys $\rightarrow$ Ser } & forward & TCATGAACTCAGCTATTCAGTCTTTGAGCAACAC \\
\hline & reverse & CTGAATAGCTGAGTTCATGAAACACGTATTT \\
\hline \multicolumn{3}{|c|}{ Primer sequences for site directed mutagenesis of USP15 histidine box } \\
\hline \multirow{2}{*}{$1^{\text {st }} \mathrm{His} \rightarrow$ Pro } & forward & ATCTGATTGCTGTTTCCAACCCCTATGGAGGGAT \\
\hline & reverse & GTTGGAAACAGCAATCAGATTATAGCGGCA \\
\hline \multirow{2}{*}{$2^{\text {nd }}$ His $\rightarrow$ Pro } & forward & ATGGAGGGATGGGAGGAGGACCCTATACTGCTTT \\
\hline & reverse & TCCTCCTCCCATCCCTCCATAGTGGTTGGA \\
\hline \multicolumn{3}{|c|}{ Primer sequences for site directed mutagenesis of USP15 aspartic acid and KRF domain } \\
\hline \multirow{2}{*}{ Asp $\rightarrow$ Gly } & forward & TCTCTGGATATCAGCAGCAAGGCTGTCAAGAACT \\
\hline & reverse & TTGCTGCTGATATCCAGAGAACTGAGGTGC \\
\hline \multirow{2}{*}{$\mathrm{KRF} \rightarrow \mathrm{NHL}$} & forward & GTACTTGTAGTACATCTCAATCAATTGTCTTACAGTCG \\
\hline & reverse & TTGAGATGTACTACAAGTACTGGAGGCAGG \\
\hline \multicolumn{3}{|c|}{ Primer sequences for semiquantitative RT-PCR } \\
\hline \multirow{2}{*}{ HPV 16 E6 } & forward & TTTCAGGACCCACAGGAGCG \\
\hline & reverse & TTACAGCTGGGTTTCTCTAC \\
\hline \multirow{2}{*}{$\beta$-actin } & forward & ACAATGAGCTGCGTGTGGCT \\
\hline & reverse & TCTCCTTAATGTCACGCACGA \\
\hline
\end{tabular}

Table 2. List of constructed mutants

\begin{tabular}{ll}
\hline Mutant number & Amino acid change \\
\hline Cysteine box mutants & $1^{\text {st }} \mathrm{Cys} \rightarrow$ Ser \\
Mutant 1 & $2^{\text {nd }}$ Cys $\rightarrow$ Ser \\
Mutant 2 & $3^{\text {rd }}$ Cys $\rightarrow$ Ser \\
Mutant 3 & $1^{\text {st }}, 2^{\text {nd }}$ Cys $\rightarrow$ Ser \\
Mutant 4 & $1^{\text {st }}, 3^{\text {rd }}$ Cys $\rightarrow$ Ser \\
Mutant 5 & $2^{\text {nd }}, 3^{\text {rd }}$ Cys $\rightarrow$ Ser \\
Mutant 6 & $1^{\text {st }}, 2^{\text {nd }}, 3^{\text {rd }}$ Cys $\rightarrow$ Ser \\
Mutant 7 & \\
\hline Histidine box mutants & $1^{\text {st }} \mathrm{His} \rightarrow$ Pro \\
Mutant 8 & $2^{\text {nd }}$ His $\rightarrow$ Pro \\
Mutant 9 & $1^{\text {st }}, 2^{\text {nd }}$ His $\rightarrow$ Pro \\
Mutant 10 & \\
\hline Aspartic acid domain mutant & Asp $\rightarrow$ Gly \\
Mutant 11 & \\
\hline KRF domain mutant & KRF $\rightarrow$ NHL \\
Mutant 12 &
\end{tabular}

FLAG precipitation was detected by anti-HPV16 E6 antibody. The controls of input were detected with corresponding antibodies, and $\beta$-actin was used as a loading control.

Semi-quantitative PCR. We used the guanidinium thiocyanate procedure for total RNA extraction by using ISOGEN (Nippon Gene, Japan). Extracted RNA was treated with RNase-free DNase under the conditions recommended by the suppliers (Boehringer Mannheim, Germany). Complementary DNA was generated from total RNA using $200 \mathrm{U}$ of the MuLV reverse transcriptase (Invitrogen) with random primers (Invitrogen). We used RTPCR for mRNA semi-quantitation. We determined the optimal number of PCR cycles required for the amplification of cDNA products to observe the predicted exponential production of the respective PCR products of HPV 16 E6 and $\beta$-actin. Amplification was carried out for 20 to 35 cycles to ensure a linear range. Finally, the relative level of HPV16 E6 mRNA was calculated by normalization with $\beta$-actin mRNA as a control, as determined by densitometry. 


\section{Results}

Identification of USP15 as a cellular target of HPV16 E6

The precise mechanism of HPV-induced carcinogenesis has not yet been fully elucidated; therefore, we attempted to identify the cellular proteins that interact with HPV 16
E6 to better understand the function of this important protein. Yeast two-hybrid screening was performed to isolate cDNAs encoding cellular proteins that interact with HPV16 E6. We screened the human fetal brain cDNA library and identified seven cDNAs that encode HPV16 E6-interacting proteins. Of these, E6-AP was previously known to interact with HPV E6 (Huibregtse et al., 1991). Another one was

(a)

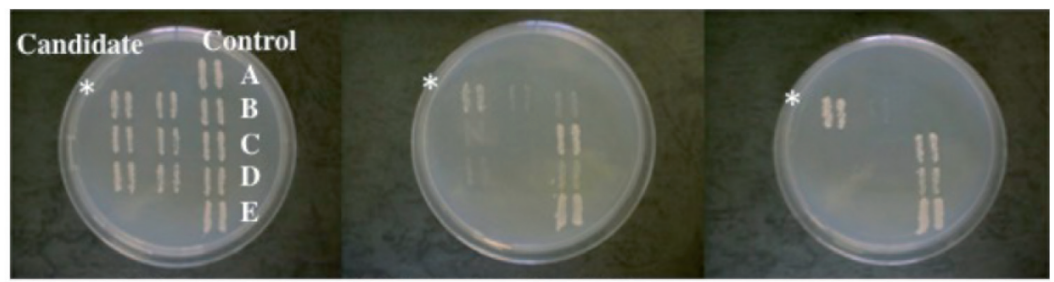

SC-Leu

SC-Leu-Trp-His + 3AT

SC-Leu-Trp-Ura

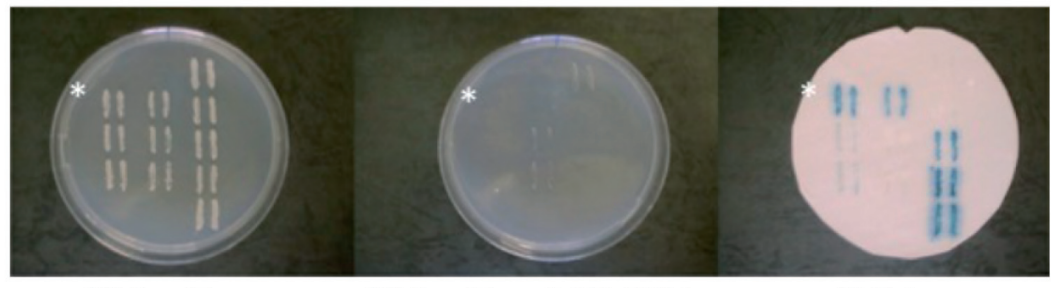

$$
\text { SC-Leu-Trp SC-Leu-Trp }+0.2 \% \text { 5FOA X-Gal }
$$

(b)

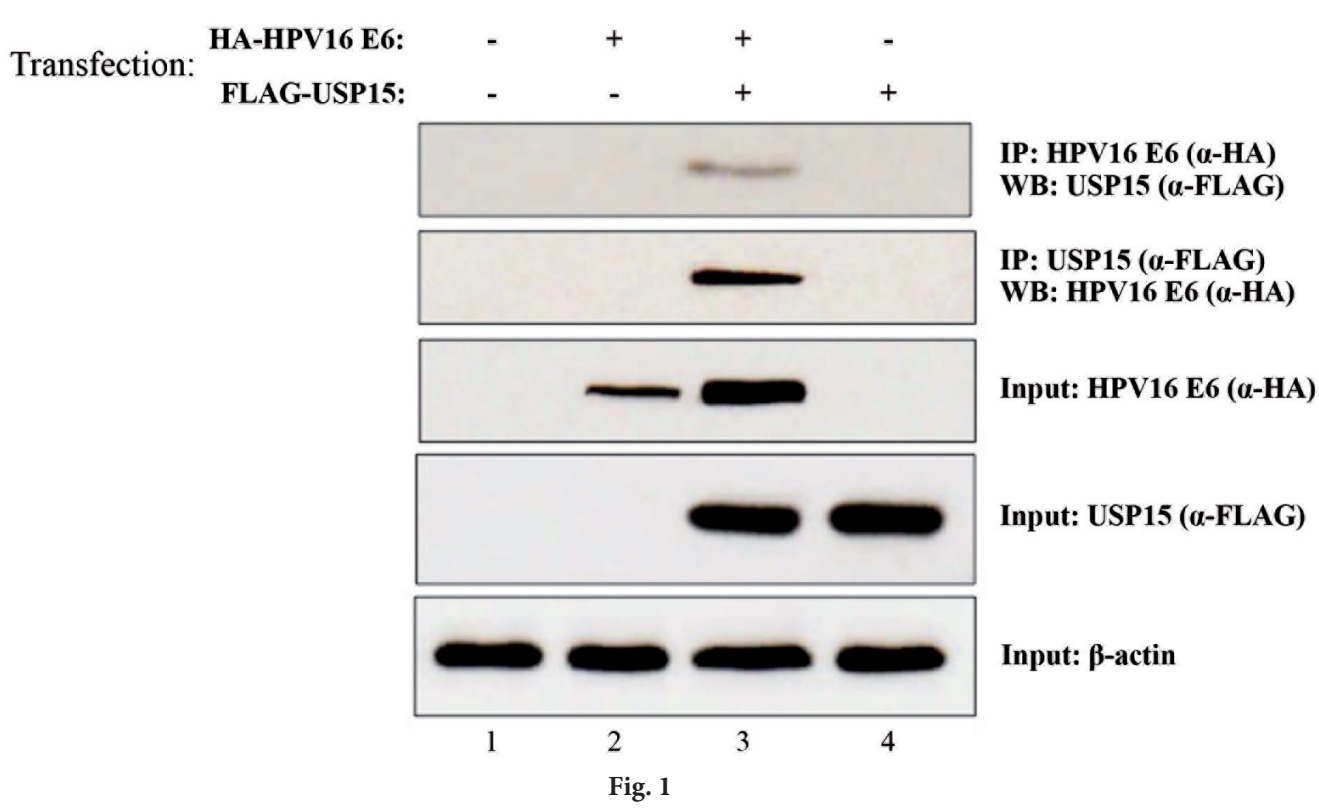

Interaction between HPV16 E6 and USP15

(a) Yeast two-hybrid assay of HPV16 E6 and USP15 $\left(^{*}\right)$ interaction. Yeast colonies containing USP15 grew on plates with SC-Leu-Trp, SC-Leu-Trp-His + $3 \mathrm{AT}$ and SC-Leu-Trp-Ura, but they did not grow on plates with SC-Leu-Trp + 0.2\% FOA. These colonies also demonstrated X-Gal activity. The SC-Leu plate was used as a control. (b) Reciprocal co-immunoprecipitation assay of HPV16 E6 interaction with USP15. 293T cells were transfected with HAtagged HPV16 E6 together with FLAG-tagged USP15. Co-immunoprecipitation with anti-HA magnetic beads was detected with anti-FLAG antibody (panel 1); with anti-FLAG M2 magnetic beads was detected with anti-HA antibody (panel 2); Western blot was analysed with anti-HA antibody (panel 3); anti-FLAG antibody (panel 4); $\beta$-actin (loading control, panel 5). Lane 1 - no trasfection; lane 2 - transfection with only pCMV-HA-HPV16 E6; lane 3 - with both pCMV-HA-HPV16 E6 and pFLAG-CMV2-USP15; lane 4 - with only pFLAG-CMV2-USP15. 
(a)

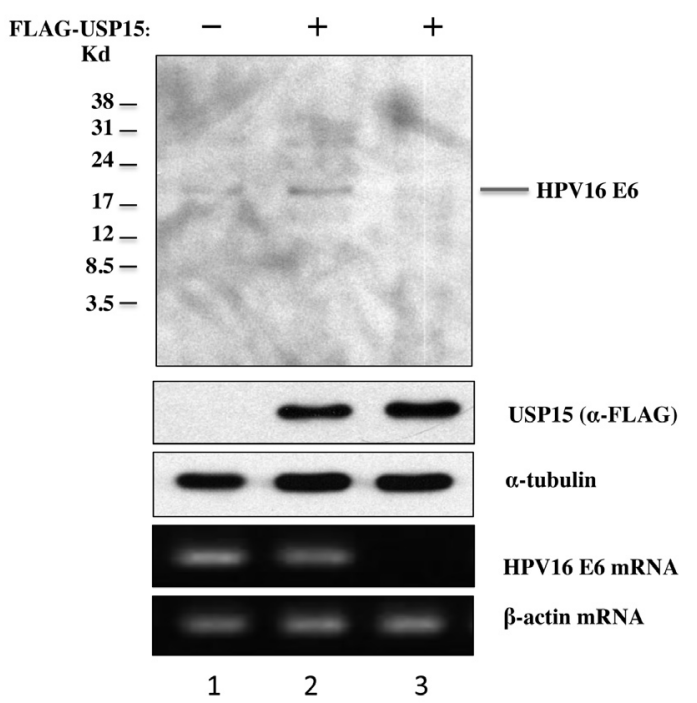

(b)

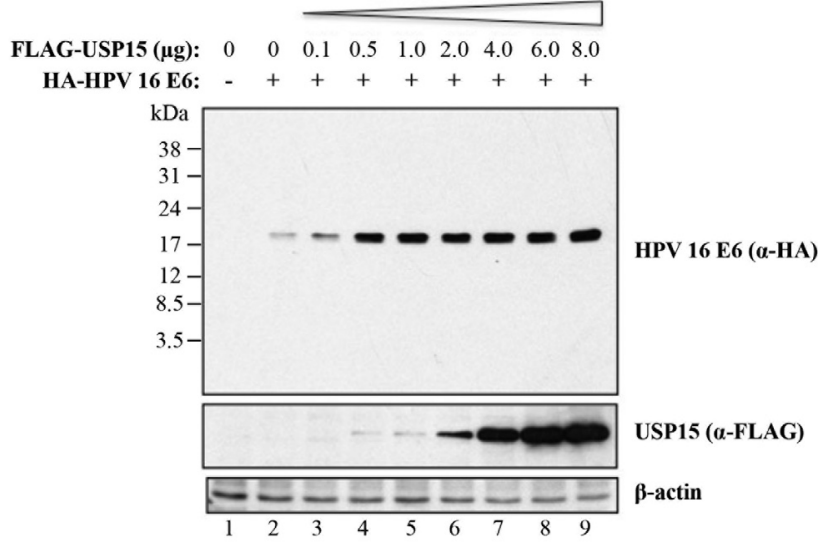

Fig. 2

Inhibition of HPV16 E6 degradation by USP15

(a) $\mathrm{SiHa}$ or $293 \mathrm{~T}$ cells were transfected with pFLAG-CMV2-USP15 and analysed by Western blot or PCR. Western blot of transfected cells was detected by anti-HPV16 E6 antibody (panel 1); anti-FLAG antibody (panel 2); anti- $\alpha$ tubulin antibody (loading control, panel 3). PCR was done to detect mRNA of HPV16 E6 (panel 4) and $\beta$-actin (loading control, panel 5). SiHa cells were transfected with empty vector (lane 1); pFLAG-CMV2-USP15 (lane 2); $293 \mathrm{~T}$ cells (no expression of HPV16) were transfected with pFLAG-CMV2-USP15 (lane 3). (b) Western blot of USP15 dose-dependent inhibition of HPV16 E6 degradation. The pFLAG-CMV2-USP15 vector was transfected into $293 \mathrm{~T}$ cells using different amounts of DNA (from 0.1 to $8.0 \mu \mathrm{g}$ ). Only $0.5 \mu \mathrm{g}$ of transfected DNA was sufficient for inhibiting the degradation of HPV16 E6. Western blot was detected with anti-HA antibody (panel 1); anti-FLAG antibody (panel 2 ) and $\beta$-actin antibody (panel 3). Cells 293T were transfected with empty vectors (lane 1); pCMV-HA-HPV16 E6 alone (lane 2); pCMV-HA-HPV16 E6 and different concentrations of pFLAG-CMV2-USP15 (lanes 3-9).

a part of the cDNA encoding USP15 (amino acids 307-962) (Fig. 1a) (Baker et al., 1999). USP15 encodes a member of the UBP family of DUBs. USP enzymes play critical roles in ubiquitin-dependent processes through polyubiquitin chain disassembly and the hydrolysis of ubiquitin-substrate bonds. In this study, because the yeast phenotypes were [SC-LeuTrp-His+3AT (+), SC-Leu-Trp+FOA (-), SC-Leu-Trp-Ura $(+)$ ] and the colonies showed X-Gal positivity (blue color), we assumed that HPV16 E6 interacts with USP15.

\section{HPV16 E6 and USP15 interaction}

To test whether HPV16 E6 physically binds to USP15 in mammalian cells, we constructed expression plasmids containing pCMV-HA-HPV16 E6 and pFLAG-CMV2USP15 and transfected them into 293 T cells. The reciprocal co-immunoprecipitation assay using anti-FLAG and anti-HA magnetic beads was performed firstly by immunoprecipitation of the cell lysates using anti-HA magnetic beads and then Western blot assay with anti-FLAG M2 antibody. Distinct specific band with appropriate size of USP15 was visualized (Fig. 1b panel 1, lane 3 ). In addition, cell lysates were also immunoprecipitated with anti-FLAG magnetic beads, and Western blot was performed using anti-HA antibody. A specific band with the size of HPV 16 E6 protein was detected (Fig. 1b, panel 2, lane 3). When only HA-HPV16 E6 or FLAG-CMV2-USP15 vector was transfected into the 293 T cells, no band was detected (Fig. 1b, lane 2 in panel 1 and lane 4 in panel 2). These results revealed that HPV16 E6 binds to USP15 in 293 T cells (Fig. 1b).

\section{USP15 inhibits the degradation of HPV16 E6}

The pCMV-HA-HPV16 E6 vector was transfected into $293 \mathrm{~T}$ cells with or without the pFLAG-CMV2-USP15 vector. It could be seen, that USP15 inhibited the degradation of HPV16 E6 when compared to the control (Fig. 1b, lane 2 and 3 in panel 3). We confirmed this effect of USP15 to endogenous HPV16 E6 using SiHa cell line (Fig. 2a). HPV16 is integrated in to the genome of SiHa cells (el Awady et al., 1987). Also, in these cells degradation of HPV 16 E6 protein was inhibited by USP15 (Fig. 2a, lane 2 in panel 1). 293T cells were transfected by FLAG-CMV2-USP15 vector as negative control (Fig. 2a, lane 3 in panel 1). As a loading control, a-tubulin was used (Fig. 2a, panel 3). Semi-quantitative PCR showed that the amount of HPV16 E6 mRNA remained mostly unchanged regardless of whether the pFLAG-CMV2USP15 vector was transfected or not (Fig. 2a, lanes 1 and 2 in panel 4). Therefore, we speculated that this degradation effect on HPV16 E6 is post-translationally regulated. As a cDNA loading control, $\beta$-actin was used (Fig. 2a, panel 5).

USP15 dose-dependently inhibits the degradation of HPV16 E6

We next investigated whether USP15 dose-dependently inhibits the degradation of HPV16 E6. Different amounts 


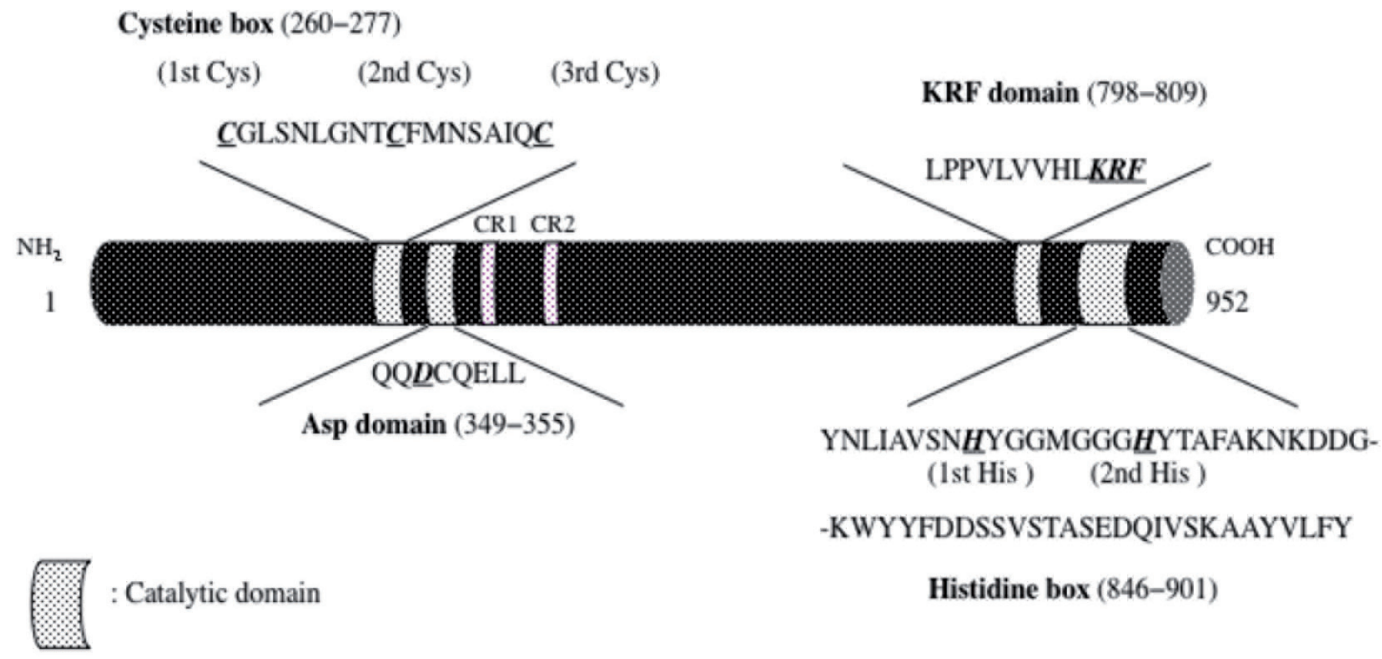

Fig. 3

\section{Schematic representation of the USP15 protein}

USP15 contains several catalytic domains, including Cys, His, Asp, and KRF boxes. Seven mutants of the Cys box, one mutant each of the Asp and KRF domains, and three mutants of the His box were constructed in this study (Table 2).

of the pFLAG-CMV2-USP15 vector (from 0.1 to $8.0 \mu \mathrm{g}$ ) were transfected into cells seeded on $10-\mathrm{cm}$ dishes. Twentyfour hours after transfection, the 293T cells were sonicated, separated on the gel and analyzed by Western blot. Even after transfection with $0.5 \mu \mathrm{g}$ of the pFLAG-CMV2-USP15 vector the USP15 protein was detectable, and the amount of HPV 16 E6 gradually increased in correlation with the amount of the pFLAG-CMV2-USP15 vector (Fig. 2b). These data show that USP15 strongly and dose-dependently inhibits the degradation of HPV16 E6.

USP15 stabilizes HPV16 E6 through its deubiquitinating enzymatic activity

USP15 contains several domains that are associated with enzymatic activity, including the Cys box, His box, Asp box, and KRF box (Fig. 3). We investigated whether disruption of these domains affects the stabilization of E6 by USP15. Several mutants of USP15 were constructed as described in Fig. 3. The Cys box of USP15 has three cysteines, and we constructed seven mutants of these portions. Each USP15 mutant of the Cys box had a reduced inhibitive effect on HPV16 E6 degradation (Fig. 4a); in particular mutant containing mutations in all three cysteines had drastically reduced (7.3\%) inhibitive effect on HPV16 E6 degradation as compared to wild-type USP15 (Fig. 4a, lane 10). The His box of USP15 has two histidines, and we constructed three His mutants. Among the His mutants, the mutation of the second histidine and both the first and second histidines had a reduced inhibitive effect on HPV16 E6 degradation
(Fig. 4b, lanes 5 and 6). We assume that in the His box, the second histidine was more important for the enzymatic activity of USP15. In addition, the Asp-Gly mutant of USP15 had a reduced inhibitive effect on HPV16 E6 degradation (Fig. 4b, lane 7). Notably, the NHL mutant of the KRF box showed drastically reduced function of USP15 as a DUB (Fig. 4b, lane 8). The NHL mutant had a reduced inhibitive effect $(3.8 \%)$ on HPV16 E6 degradation as compared to wild-type USP15. These data demonstrate that catalytically inactive USP15 has reduced function as a DUB for HPV 16 E6, therefore, we confirmed that USP15 stabilizes HPV16 E6 through its deubiquitinating enzymatic activity.

\section{Discussion}

High-risk HPVs such as HPV16 and HPV18, initiate the development of multiple types of cancers in the anogenitalia, including essentially $100 \%$ of cervical cancers (zur Hausen, 2000). The high-risk HPV types encode two oncoproteins, E6 and E7. A key event of HPV-induced oncogenesis is the integration of viral DNA into the human genome. Integration results in the disruption of the viral E2 DNA region, inducing the over-expression of the viral E6 and E7 oncoproteins and promoting their oncogenic functions effectively in the infected human cells (Eichten et al., 2004). The high-risk HPV E6 protein plays crucial roles in high-risk HPV-induced cancers. HPV E6 is a transcriptional transactivator that binds double-stranded DNA. HPV E6 proteins are approximately 150 residues long and they contain two zinc finger motifs composed of four C- 
(a)

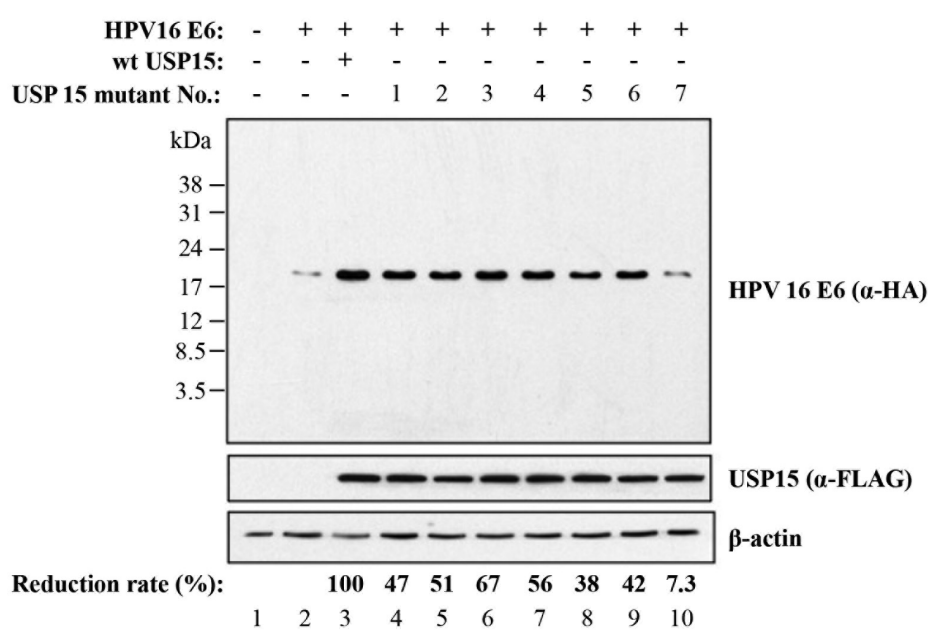

(b)

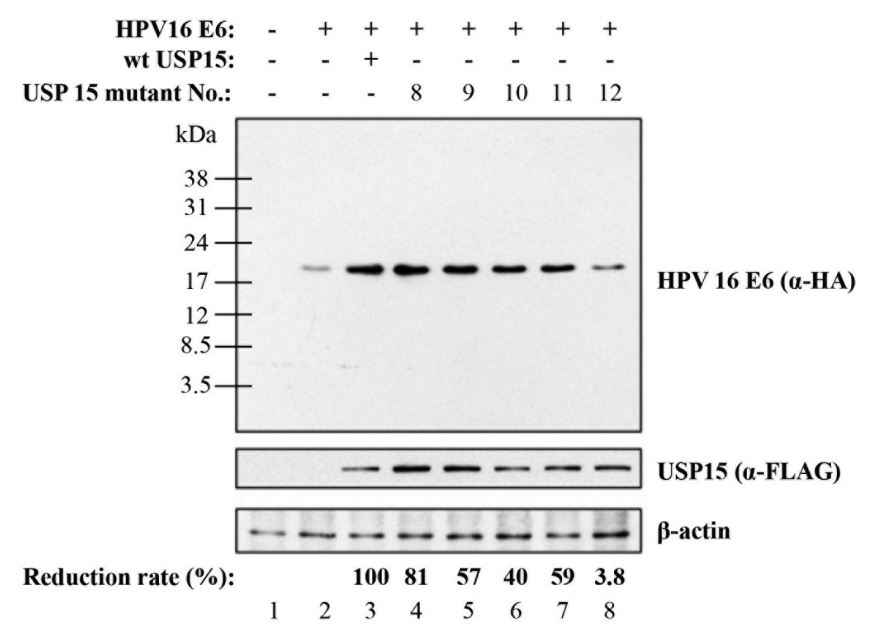

Fig. 4

Analysis of the function of catalytically inactive USP15 mutants to HPV16 E6

Western blot of USP15 mutants 1-7 (a) and 8-12 (b) was analysed by: anti-HA antibody (panel 1); anti-FLAG antibody (panel 2) and anti $\beta$-actin antibody (loading control, panel 3). The reduction rate (\%) of HPV16 E6 degradation was calculated by using NIH Image. All USP15 mutants (1-12) had a reduced inhibitive effect on HPV16 E6 degradation. In particular, mutants 7 and 12 showed reduction of up to $7.3 \%$ and 3.8\% respectively. Cells $293 \mathrm{~T}$ were transfected with empty vectors (lane 1); pCMV-HA-HPV16 E6 alone (lane 2); pCMV-HA-HPV16 E6 and wild type pFLAG-CMV2-USP15 (lane 3); 1-7 mutants of pFLAG-CMV2-USP15 ((a) lane 4-10); 8-12 mutants of pFLAG-CMV2-USP15 ((b) lane 4-8).

X-X-C motifs that are required for E6 function (Barbosa et al., 1989; Barbosa and Schlegel, 1989; Cole and Danos, 1987). HPV E6 not only binds to E6-AP to form a complex that targets the p53 tumor suppressor protein for proteasome-mediated degradation, but it also impedes $\mathrm{p} 53$ acetylation by hindering the inactivation of $\mathrm{p} 53$ and inhibitor of growth protein 4 (ING4) (Guo et al., 2013). HPV E6 directly interacts with the hTERT protein to facilitate the post-translational modification of hTERT, which enhances the activity of hTERT (Van Doorslaer and Burk, 2012). It also has transforming activity that inactivates the human $\mathrm{p} 53$ tumor suppressor protein by targeting it for proteasome degradation. The HPV E6 oncoprotein is involved in cell cycle deregulation, where p53 is abrogated. Although the high-risk HPV E6 has important functions in high-risk $\mathrm{HPV}$-induced cancers, these functions remain poorly understood. Therefore, we attempted to identify the proteins that interact with HPV16 E6 to understand the specific function of HPV16 E6. In this report, we confirmed USP15 as a protein that interacts with HPV16 E6, because USP15 has already been reported to associate with HPV16 E6 in a mass spectrometry analysis in 2009 (Vos et al., 2009). Here, we used the yeast twohybrid system to show that the USP15 protein binds to HPV16 E6, resulting in a positive interaction. We also showed for the first time that USP15 stabilizes the HPV16 E6 protein through 
deubiquitination, which ultimately leads to the inhibition of HPV16 E6 degradation. DUBs affect the ubiquitin status of target proteins. The degradation of proteins is an irreversible process, so specificity in the ubiquitin system must be strictly maintained. USPs can remove covalently attached ubiquitin moieties from target proteins and regulate both the stability and ubiquitin-signaling state of their substrates. Aberrations in the ubiquitin-proteasome system have been recently connected to the pathogenesis of several human protein degradation disorders, including cancer and neurodegenerative diseases. As such, the proteasome is now considered to be important in the mechanism of carcinogenesis (Chen and Zhao, 2013; Jarvius et al., 2013; Yang et al., 2009).

In this study, we showed that USP15 could not induce HPV16 E6 transcription; therefore, we speculated that HPV16 E6 is regulated post-translationally by USP15. But Vos et al. (2009) reported that USP15 induced HPV16 E6 transcripts. Further analysis will be needed at this point. USP15 is a ubiquitously expressed DUB that was first discovered in 1999; it belongs to the USP family of DUBs (Baker et al., 1999). Since the discovery of USP15, not much has been revealed about its function nor the association between USP15 and cancer. Only a few studies have reported data on the association between carcinogenesis and USP15. USP15 mediates the stability of MDM2 in cancers (Zou et al., 2014), and because USP15 binds to MDM2 and cleaves the ubiquitin chains from MDM2, USP15 functions as a deubiquitinase of MDM2. USP15 was reported to be overexpressed as a result of USP15 gene amplification in some glioblastomas (Eichhorn et al., 2012), and USP15 gene amplification was also present in breast and ovarian cancers (Eichhorn et al., 2012), indicating that the oncogenic role of USP15 is associated with the carcinogenesis of some human cancers (Zou et al., 2014).

In the present study, we confirmed that HPV16 E6 interacted with USP15 and inhibited the degradation of HPV 16 E6. Protein degradation via the ubiquitin system plays a crucial role in numerous cellular signaling pathways and so does USP15 as one of the DUBs. USP15 has been identified as a gene that encodes a protein of 952 amino acids that contains the highly conserved Cys and His boxes present in all members of the UBP family of DUBs (Baker et al., 1999). We showed that disruptions of the Cys, His, Asp, and KRF boxes of USP15 result in reduced inhibition of HPV16 E6 degradation. In particular, Cys box and KRF domain of USP15 were important for the inhibition of HPV16 E6 degradation. USP15, but not the catalytically inactive USP15 mutants, was capable of inhibiting the degradation of HPV16 E6.

After HPV16 infects the human cells, the viral oncoprotein E6 is expressed in the cells. Subsequently, the HPV16 E6 protein binds USP15, which is produced by human cells, and the degradation of HPV16 E6 is inhibited, thus demonstrating the function of E6 as an oncoprotein. The function of the E6 protein may be seen in the progression to cervical intraepithelial neoplasia (CIN) 1 and 2 at the episomal state, and then to CIN3 after the integration of HPV DNA into human chromosomes (Briolat et al., 2007; Cricca et al., 2007; Evans et al., 2008; Hafner et al., 2008; Hopman et al., 2004; Vinokurova et al., 2008). USP15 appears to exert its effects on HPV16 E6 by influencing protein stability. From these data, we hypothesized that USP15 inhibits the degradation of HPV16 E6 by binding each other immediately after the infection in human cells. In CIN1 and 2, the HPV DNA is maintained in the undifferentiated basal cells of the squamous cell layer as extrachromosomal nuclear plasmids, and HPV16 E6 expression is relatively suppressed. HPV16 E6 is induced by the suppression of HPV E2 due to disruption of the HPV DNA that causes integration into human chromosomes. This induction of HPV E6 by the suppression of HPV E2 expression is observed in CIN3, as the integration of HPV DNA into human chromosomes was seen at this stage (CIN3), and USP15 inhibited the degradation of HPV16 E6 in these lesions. Therefore, USP15 is very important for the maintenance of HPV16 E6 via the inhibition of HPV16 E6 degradation even in normal cervical squamous cells and in all the intraepithelial lesions.

We conclude that the inhibition of HPV16 E6 degradation by USP15 is important for its function as an oncoprotein in cervical carcinogenesis. Our results confirmed a potentially significant interaction between HPV16 E6 and USP15 as Vos et al. already reported, because these data suggest that agents that antagonize USP15 may be therapeutically useful for preventing disease onset and progression through HPV16 E6 stabilization.

Acknowledgments. This study was funded by the donation of Asahikawa Medical University Alumni Association, and the Department of Obstetrics \& Gynecology Alumni Association (Asahikawa Medical University) and the member of the Japanese Society of Pathology (Hokkaido branch).

\section{References}

Alarcon R, Koumenis C, Geyer RK, Maki CG, Giaccia AJ (1999): Hypoxia induces p53 accumulation through MDM2 down-regulation and inhibition of E6-mediated degradation. Cancer Res. 59, 6046-6051.

Ansari T, Brimer N, Vande Pol SB (2012): Peptide interactions stabilize and restructure human papillomavirus type 16 E6 to interact with p53. J. Virol. 86, 11386-11391. https:// doi.org/10.1128/JVI.01236-12

Baker RT, Tobias JW, Varshavsky A (1992): Ubiquitin-specific proteases of Saccharomyces cerevisiae. Cloning of UBP2 and UBP3, and functional analysis of the UBP gene family. J. Biol. Chem. 267, 23364-23375.

Baker RT, Wang XW, Woollatt E, White JA, Sutherland GR (1999): Identification, functional characterization, and chromosomal localization of USP15, a novel human ubiquitin-specific protease related to the UNP oncoprotein, and a systematic nomenclature for human ubiquitin- 
specific proteases. Genomics 59, 264-274. https://doi. org/10.1006/geno.1999.5879

Barbosa MS, Lowy DR, Schiller JT (1989): Papillomavirus polypeptides E6 and E7 are zinc-binding proteins. J. Virol. 63, 1404-1407.

Barbosa MS, Schlegel R (1989): The E6 and E7 genes of HPV-18 are sufficient for inducing two-stage in vitro transformation of human keratinocytes. Oncogene 4, 1529-1532.

Beaudenon S, Huibregtse JM (2008): HPV E6, E6AP and cervical cancer. BMC Biochem. 9 (Suppl. 1), S4. https://doi. org/10.1186/1471-2091-9-S1-S4

Briolat J, Dalstein V, Saunier M, Joseph K, Caudroy S, Pretet JL, Birembaut P, Clavel C (2007): HPV prevalence, viral load and physical state of HPV-16 in cervical smears of patients with different grades of CIN. Int. J. Cancer 121, 2198-2204. https://doi.org/10.1002/ijc.22959

Chen FZ, Zhao XK (2013): Ubiquitin-proteasome pathway and prostate cancer. Onkologie 36, 592-596. https://doi. org/10.1159/000355166

Clague MJ, Coulson JM, Urbe S (2012): Cellular functions of the DUBs. J. Cell Sci. 125, 277-286. https://doi.org/10.1242/ jcs.090985

Cole ST, Danos O (1987): Nucleotide sequence and comparative analysis of the human papillomavirus type 18 genome. Phylogeny of papillomaviruses and repeated structure of the E6 and E7 gene products. J. Mol. Biol. 193, 599-608. https://doi.org/10.1016/0022-2836(87)90343-3

Cooper B, Schneider S, Bohl J, Jiang Y, Beaudet A, Vande Pol S (2003): Requirement of E6AP and the features of human papillomavirus E6 necessary to support degradation of p53. Virology 306, 87-99. https://doi.org/10.1016/S0042$\underline{6822(02) 00012-0}$

Cricca M, Morselli-Labate AM, Venturoli S, Ambretti S, Gentilomi GA, Gallinella G, Costa S, Musiani M Zerbini M (2007): Viral DNA load, physical status and E2/E6 ratio as markers to grade HPV16 positive women for high-grade cervical lesions. Gynecol. Oncol. 106, 549-557. https:// doi.org/10.1016/j.ygyno.2007.05.004

Crook T, Fisher C, Masterson PJ, Vousden KH (1994): Modulation of transcriptional regulatory properties of $\mathrm{p} 53$ by HPV E6. Oncogene 9, 1225-1230.

Crook T, Tidy JA, Vousden KH (1991): Degradation of p53 can be targeted by HPV E6 sequences distinct from those required for $\mathrm{p} 53$ binding and trans-activation. Cell 67, 547556. https://doi.org/10.1016/0092-8674(91)90529-8

Dell G, Gaston K (2001): Human papillomaviruses and their role in cervical cancer. Cell Mol. Life Sci. 58, 1923-1942. https:// doi.org/10.1007/PL00000827

Eichhorn PJ, Rodon L, Gonzalez-Junca A, Dirac A, Gili M, Martinez-Saez E, Aura C, Barba I, Peg V, Prat A, Cuartas I, Jimenez J, Garcia-Dorado D, Sahuquillo J, Bernards R, Baselga J, Seoane J (2012): USP15 stabilizes TGF-beta receptor I and promotes oncogenesis through the activation of TGF-beta signaling in glioblastoma. Nat. Med. 18, 429-435. https://doi.org/10.1038/nm.2619

Eichten A, Rud DS, Grace M, Piboonniyom SO, Zacny V, Munger $\mathrm{K}$ (2004): Molecular pathways executing the "trophic sentinel" response in HPV-16 E7-expressing normal human dip- loid fibroblasts upon growth factor deprivation. Virology 319, 81-93. https://doi.org/10.1016/j.virol.2003.11.008

el Awady MK, Kaplan JB, O'Brien SJ, Burk RD (1987): Molecular analysis of integrated human papillomavirus 16 sequences in the cervical cancer cell line SiHa. Virology 159, 389398. https://doi.org/10.1016/0042-6822(87)90478-8

Evans MF, Adamson CS, Cooper K (2008): Evidence of HPV16 integration in low- and high-grade cervical lesions that regress demonstrated by multiple displacement amplification and Southern blot hybridisation. J. Clin. Pathol. 61, 541-543. https://doi.org/10.1136/jcp.2007.051797

Guo Y, Meng X, Wang Q, Wang Y, Shang H (2013): The ING4 binding with $\mathrm{p} 53$ and induced $\mathrm{p} 53$ acetylation were attenuated by human papillomavirus 16 E6. PLoS One 8, e71453. https://doi.org/10.1371/journal.pone.0071453

Hafner N, Driesch C, Gajda M, Jansen L, Kirchmayr R, Runnebaum IB, Durst M (2008): Integration of the HPV16 genome does not invariably result in high levels of viral oncogene transcripts. Oncogene 27, 1610-1617. https:// doi.org/10.1038/sj.onc. 1210791

Hopman AH, Smedts F, Dignef W, Ummelen M, Sonke G, Mravunac M, Vooijs GP, Speel EJ, Ramaekers FC (2004): Transition of high-grade cervical intraepithelial neoplasia to micro-invasive carcinoma is characterized by integration of HPV 16/18 and numerical chromosome abnormalities. J. Pathol. 202, 23-33. https://doi.org/10.1002/path.1490

Howie HL, Koop JI, Weese J, Robinson K, Wipf G, Kim L, Galloway DA (2011): Beta-HPV 5 and 8 E6 promote p300 degradation by blocking AKT/p300 association. PLoS Pathog. 7, e1002211. https://doi.org/10.1371/journal.ppat.1002211

Huibregtse JM, Scheffner M, Howley PM (1991): A cellular protein mediates association of 553 with the E6 oncoprotein of human papillomavirus types 16 or 18. EMBO J. 10, 4129-4135.

Jarvius M, Fryknas M, D'Arcy P, Sun C, Rickardson L, Gullbo J, Haglund C, Nygren P, Linder S, Larsson R (2013): Piperlongumine induces inhibition of the ubiquitinproteasome system in cancer cells. Biochem. Biophys. Res. Commun. 431, 117-123. https://doi.org/10.1016/j. bbrc.2013.01.017

Kawakami T, Suzuki T, Baek SH, Chung CH, Kawasaki H, Hirano H, Ichiyama A, Omata M, Tanaka K (1999): Isolation and characterization of cytosolic and membrane-bound deubiquitinylating enzymes from bovine brain. J. Biochem. 126, 612-623. https://doi.org/10.1093/oxfordjournals. jbchem.a022493

Kehmeier E, Ruhl H, Voland B, Stoppler MC, Androphy E, Stoppler H (2002): Cellular steady-state levels of "high risk" but not "low risk" human papillomavirus (HPV) E6 proteins are increased by inhibition of proteasome-dependent degradation independent of their p53- and E6AP-binding capabilities. Virology 299, 72-87. https://doi.org/10.1006/viro.2002.1502

Kim SY, Lee JH, Yang ES, Kil IS, Park JW (2003): Human sensitive to apoptosis gene protein inhibits peroxynitrite-induced DNA damage. Biochem. Biophys. Res. Commun. 301, 671-674. https://doi.org/10.1016/S0006-291X(03)00018-4

Li X, Coffino, P (1996): High-risk human papillomavirus E6 protein has two distinct binding sites within p53, of which only one determines degradation. J. Virol. 70, 4509-4516. 
Liu X, Roberts J, Dakic A, Zhang Y, Schlegel R (2008): HPV E7 contributes to the telomerase activity of immortalized and tumorigenic cells and augments E6-induced hTERT promoter function. Virology 375, 611-623. https://doi. org/10.1016/j.virol.2008.02.025

Oh ST, Longworth MS, Laimins LA (2004): Roles of the E6 and E7 proteins in the life cycle of low-risk human papillomavirus type 11. J. Virol. 78, 2620-2626. https://doi.org/10.1128/ JVI.78.5.2620-2626.2004

Pim D, Banks L (1999): HPV-18 E6 ${ }^{\star}$ I protein modulates the E6directed degradation of $\mathrm{p} 53$ by binding to full-length HPV-18 E6. Oncogene 18, 7403-7408. https://doi. org/10.1038/sj.onc.1203134

Scheffner M, Huibregtse JM, Vierstra RD, Howley PM (1993): The HPV-16 E6 and E6-AP complex functions as a ubiquitinprotein ligase in the ubiquitination of p53. Cell 75, 495505. https://doi.org/10.1016/0092-8674(93)90384-3

Schwarz E, Freese UK, Gissmann L, Mayer W, Roggenbuck B, Stremlau A, zur Hausen H (1985): Structure and transcription of human papillomavirus sequences in cervical carcinoma cells. Nature 314, 111-114. https://doi. org $/ 10.1038 / 314111 \mathrm{a} 0$

Sherman L, Itzhaki H, Jackman A, Chen JJ, Koval D, Schlegel R (2002): Inhibition of serum- and calcium-induced terminal differentiation of human keratinocytes by HPV 16 E6: study of the association with p53 degradation, inhibition of p53 transactivation, and binding to E6BP. Virology 292, 309-320. https://doi.org/10.1006/viro.2001.1263

Stewart D, Kazemi S, Li S, Massimi P, Banks L, Koromilas AE, Matlashewski G (2004): Ubiquitination and proteasome degradation of the E6 proteins of human papillomavirus types 11 and 18. J. Gen. Virol. 85, 1419-1426. https://doi. org/10.1099/vir.0.19679-0

Strohl AE, Mendoza G, Ghant MS, Cameron KA, Simon MA, Schink JC, Marsh EE (2015): Barriers to prevention: knowledge of HPV, cervical cancer, and HPV vaccinations among African American women. Am. J. Obstet. Gynecol. 212, 65 e1-5. https://doi.org/10.1016/j.ajog.2014.06.059

Thomas M, Pim D, Banks L (1999): The role of the E6-p53 interaction in the molecular pathogenesis of HPV. Oncogene 18, 7690-7700. https://doi.org/10.1038/sj.onc. 1202953

Van Doorslaer K, Burk RD (2012): Association between hTERT activation by HPV E6 proteins and oncogenic risk. Virology 433, 216-219. https://doi.org/10.1016/j. virol.2012.08.006

Vande Pol SB, Brown MC, Turner CE (1998): Association of bovine papillomavirus type 1 E6 oncoprotein with the focal adhesion protein paxillin through a conserved protein interaction motif. Oncogene 16, 43-52. https:// doi.org/10.1038/sj.onc.1201504

Vinokurova S, Wentzensen N, Kraus I, Klaes R, Driesch C, Melsheimer P, Kisseljov F, Durst M, Schneider A, von Knebel Doeberitz M (2008): Type-dependent integration frequency of human papillomavirus genomes in cervical lesions. Cancer Res 68, 307-313. https://doi. org/10.1158/0008-5472.CAN-07-2754

Vos, RM, Altreuter, J, White, EA, and Howley, PM (2009): The ubiquitin-specific peptidase USP15 regulates human papillomavirus type 16 E6 protein stability. J. Virol. 83, 8885-8892. https://doi.org/10.1128/JVI.00605-09

Wang HY, Kim G, Cho H, Kim S, Lee D, Park S, Park KH, Lee H (2015a): Diagnostic performance of HPV E6/E7, hTERT, and Ki67 mRNA RT-qPCR assays on formalin-fixed paraffin-embedded cervical tissue specimens from women with cervical cancer. Exp. Mol. Pathol. 98, 510-516. https://doi.org/10.1016/j.yexmp.2015.03.036

Wang HY, Park S, Kim S, Lee D, Kim G, Kim Y, Park KH, Lee H (2015b): Use of hTERT and HPV E6/E7 mRNA RTqPCR TaqMan assays in combination for diagnosing high-grade cervical lesions and malignant tumors. Am. J. Clin. Pathol. 143, 344-351. https://doi.org/10.1309/ AJCPF2XGZ2XIQYQX

White EA, Kramer RE, Tan MJ, Hayes SD, Harper JW, Howley PM (2012): Comprehensive analysis of host cellular interactions with human papillomavirus E6 proteins identifies new E6 binding partners and reflects viral diversity. J. Virol. 86, 13174-13186. https://doi.org/10.1128/ JVI.02172-12

Wu DW, Chuang CY, Lin WL, Sung WW, Cheng YW, Lee H (2014): Paxillin promotes tumor progression and predicts survival and relapse in oral cavity squamous cell carcinoma by microRNA-218 targeting. Carcinogenesis 35, 1823-1829. https://doi.org/10.1093/carcin/bgu102

Xie X, Piao L, Bullock BN, Smith A, Su T, Zhang M, Teknos TN, Arora PS, Pan Q (2014): Targeting HPV16 E6-p300 interaction reactivates $\mathrm{p} 53$ and inhibits the tumorigenicity of HPV-positive head and neck squamous cell carcinoma. Oncogene 33, 1037-1046. https://doi.org/10.1038/ onc. 2013.25

Xu M, Katzenellenbogen RA, Grandori C, Galloway DA (2013): An unbiased in vivo screen reveals multiple transcription factors that control HPV E6-regulated hTERT in keratinocytes. Virology 446, 17-24. https://doi.org/10.1016/j. virol.2013.07.014

Yang X, Lu L (2015): Expression of HPV-16 E6 protein and p53 inactivation increases the uterine cervical cancer invasion. Drug. Res. (Stuttg.) 65, 70-73.

Yang Y, Kitagaki J, Wang H, Hou DX, Perantoni AO (2009): Targeting the ubiquitin-proteasome system for cancer therapy. Cancer. Sci. 100, 24-28. https://doi.org/10.1111/j.13497006.2008.01013.x

Zou Q, Jin J, Hu H, Li HS, Romano S, Xiao Y, Nakaya M, Zhou X, Cheng X, Yang P, Lozano G, Zhu C, Watowich SS, Ullrich SE, Sun SC (2014): USP15 stabilizes MDM2 to mediate cancer-cell survival and inhibit antitumor $\mathrm{T}$ cell responses. Nat. Immunol. 15, 562-570. https://doi. org/10.1038/ni.2885

zur Hausen H (1991a): Human papillomaviruses in the pathogenesis of anogenital cancer. Virology 184, 9-13. https://doi. org/10.1016/0042-6822(91)90816-T

zur Hausen H (1991b): Viruses in human cancers. Science 254, 1167-1173. https://doi.org/10.1126/science. 1659743

zur Hausen H (2000): Papillomaviruses causing cancer: evasion from host-cell control in early events in carcinogenesis. J. Natl. Cancer. Inst. 92, 690-698. https://doi.org/10.1093/ inci/92.9.690 